\title{
Effect of Slit/Robo signaling on regeneration in lung emphysema
}

\author{
Jin-Soo Park', RyeonJin Cho', Eun-Young Kang ${ }^{1}$ and Yeon-Mok Oh' ${ }^{2,3}$
}

\begin{abstract}
Emphysema, a pathological component of chronic obstructive pulmonary disease, causes irreversible damage to the lung. Previous studies have shown that Slit plays essential roles in cell proliferation, angiogenesis, and organ development. In this study, we evaluated the effect of Slit2 on the proliferation and migration of mouse lung epithelial cells and its role in regeneration in an emphysema lung mouse model. Here, we have shown that Slit2/Robo signaling contributes to the regeneration of lungs damaged by emphysema. Mouse epithelial lung cells treated with Slit2 exhibited increased proliferation and migration in vitro. Our results also showed that Slit2 administration improved alveolar regeneration in the emphysema mouse model in vivo. Furthermore, Slit2/Robo signaling increased the phosphorylation of ERK and Akt, which was mediated by Ras activity. These Slit2-mediated cellular signaling processes may be involved in the proliferation and migration of mouse lung epithelial cells and are also associated with the potential mechanism of lung regeneration. Our findings suggest that Slit2 administration may be beneficial for alveolar regeneration in lungs damaged by emphysema.
\end{abstract}

\section{Introduction}

Chronic obstructive pulmonary disease (COPD) is characterized by both chronic bronchiolitis and emphysema, with a high global rate of morbidity and mortality ${ }^{1,2}$. To target chronic bronchiolitis, various medications, such as bronchodilators or those with anti-inflammatory effects, have been developed over the past 20 years. However, no treatment has thus far been capable of successfully inducing regeneration in lungs with emphysema, which damages lung alveoli. Slit/Robo signaling, a well-known mediator of nervous system development ${ }^{3,4}$, has also been implicated in cell proliferation, stem cell regulation, angiogenesis, and organ development ${ }^{5-9}$. Recent research has also indicated that Slit/Robo signaling could have important functions in the reproductive system $^{10}$.

\footnotetext{
Correspondence: Yeon-Mok Oh (ymoh55@amc.seoul.kr)

${ }^{1}$ Asan Institute for Life Sciences, Seoul, Korea

${ }^{2}$ Department of Internal Medicine, University of Ulsan College of Medicine, Seoul, Korea

Full list of author information is available at the end of the article
}

Slits are secreted extracellular matrix proteins that bind to the Roundabout (Robo) receptor ${ }^{11,12}$. Three Slit protein isoforms (Slit1-3) have been identified in vertebrates and are characterized by four leucine-rich repeat domains, seven to nine epidermal growth factor repeat domains, a laminin G domain, and a C-terminal cysteine-rich domain $^{3,13,14}$.

Four Robo receptors (Robo1-4) consist of five immunoglobulin (Ig)-like domains with three fibronectin (FN) type III-like domains, a single transmembrane domain, and a long 457 amino acid cytoplasmic tail ${ }^{13-15}$.

Given its role in neurogenesis, cell proliferation, and migration, we hypothesized that Slit/Robo signaling may facilitate regeneration in lungs damaged by emphysema. To test this hypothesis, we evaluated the regenerative effects of the Slit protein in both mouse lung epithelial cells and an elastase-induced emphysema mouse model. Cell proliferation and migration were increased in lung epithelial cells treated with Slit2. Histological analysis and measurement of lung emphysema severity with mean linear intercepts (MLIs) showed that intranasal administration of Slit2 improved lung alveolar regeneration in the

\section{(c) The Author(s) 2021}

(c) (i) Open Access This article is licensed under a Creative Commons Attribution 4.0 International License, which permits use, sharing, adaptation, distribution and reproduction cc) in any medium or format, as long as you give appropriate credit to the original author(s) and the source, provide a link to the Creative Commons license, and indicate if changes were made. The images or other third party material in this article are included in the article's Creative Commons license, unless indicated otherwise in a credit line to the material. If material is not included in the article's Creative Commons license and your intended use is not permitted by statutory regulation or exceeds the permitted use, you will need to obtain permission directly from the copyright holder. To view a copy of this license, visit http://creativecommons.org/licenses/by/4.0/. 
emphysema mouse model. These results suggest that Slit2 may have the potential as a new drug candidate for the regeneration of lungs damaged by emphysema.

\section{Materials and methods}

\section{Epithelial cell proliferation and migration in vitro}

Cell proliferation analysis was performed on mouse lung epithelial (MLE-12) cells using Cell Counting Kit-8 (CCK8, Enzo). MLE-12 cells were seeded on cell culture plates and treated with different concentrations of recombinant murine Slit2 protein (Slit2, R\&D Systems). After each time point, proliferation was measured by CCK- 8 assay in accordance with the manufacturer's protocol.

Cell migration was analyzed using a scratch wound healing assay, which involved using a T1000 pipette tip on monolayers of MLE-12 cell cultures. Cells were then incubated in culture media with 50, 100, 200, and $500 \mathrm{ng}$ Slit2 at $37^{\circ} \mathrm{C}$. After 0,72 , and $120 \mathrm{~h}$ of incubation, the gap width of the repopulated scratch was measured and recorded and then compared with the initial gap size at $0 \mathrm{~h}$.

\section{Quantitative real-time polymerase chain reaction (RT-PCR)}

Total RNA was isolated from cells or whole-lung tissue using the RNeasy Plus Mini Kit (Qiagen) and synthesized into cDNA using a Maxima First Strand cDNA synthesis kit (Thermo Scientific). Quantitative RT-PCR analyses were performed with a real-time LightCycler 480 and SYBR Green I Master mix.

\section{Mice}

Female C57BL/6 mice, aged 7 weeks, were purchased from Orient Bio (Seongnam, Korea) and maintained under specific pathogen-free conditions in the animal facility of the Asan Institute for Life Sciences. This experiment was approved by the Institutional Animal Care and Use Committee of Asan Medical Center (IACUC. 2017-13-274).

\section{Elastase-induced lung emphysema model}

For the experimental emphysema model, we generated elastase-induced model mice using previously described methods ${ }^{16}$. The mice were intratracheally injected with porcine pancreatic elastase (PPE, Sigma-Aldrich, St. Louis, MO, USA) on day 0 . The mice were treated with Slit2 by intranasal administration on days 7-13 and were sacrificed on day 14 , after which the lungs were harvested.

\section{Histology and quantification of emphysema}

Lung tissue was inflated with $0.5 \%$ low-melting agarose, fixed with $4 \%$ formalin, and embedded in paraffin. Lung sections with a thickness of $6 \mu \mathrm{m}$ were stained with hematoxylin and eosin. The severity of emphysema was measured by MLIs. The MLIs of the lung tissue sections were determined using previously described methods ${ }^{17}$.

\section{Slit2/Robo binding ELISA}

A 96-well microplate was coated with $50 \mu \mathrm{L}$ of $10 \mu \mathrm{g}$ of Slit2 or bovine serum albumin (BSA) per well and incubated overnight at $4{ }^{\circ} \mathrm{C}$. The coated plate was then blocked with $100 \mu \mathrm{L}$ of phosphate-buffered saline (PBS) containing $1 \%$ BSA per well for $1 \mathrm{~h}$ at room temperature. The plates were washed three times with $200 \mu \mathrm{L}$ of $0.05 \%$ Tween-20 diluted in PBS. MLE-12 cell lysates $(100 \mu \mathrm{L} /$ well) were added to the coated wells, and the plates were incubated for $1 \mathrm{~h}$ at room temperature. After being washed, the Robo antibody (1:1000) was added to the 96well microplates and incubated for $1.5 \mathrm{~h}$ at room temperature. After being washed, horseradish peroxidase (HRP)-conjugated secondary antibody was added and incubated for $1 \mathrm{~h}$ at room temperature. TMB $\left(3,3^{\prime}, 5,5^{\prime}\right.$ tetramethylbenzidine) substrate solution was added to the 96-well microplates to induce a color reaction. The reaction was then stopped with a stop solution $(0.18 \mathrm{M}$ sulfuric acid). The plate was measured at $405 \mathrm{~nm}$ on a microplate reader (BioRad).

\section{siRNA transfection}

Robo1 siRNA (Bioneer) was transfected into MLE-12 cells with $100 \mathrm{nmol}$ siRNA in a 6-well plate with Lipofectamine RNAiMax (Invitrogen) according to the manufacturer's instructions. After $4 \mathrm{~h}$ of transfection, the cell culture media was replaced with fresh media, and the cells were used for experiments $48 \mathrm{~h}$ after transfection.

\section{GTPase activity assay}

For active GTPase analysis, MLE-12 cells were incubated alone or with Slit2, and the cells were collected at $15 \mathrm{~m}, 1 \mathrm{~h}$, and $6 \mathrm{~h}$. To harvest the cells, the media was removed, and cells were rinsed with cold PBS. The cells were then resuspended in $0.5 \mathrm{~mL}$ of ice-cold $1 \times$ lysis/binding/wash buffer plus $1 \mathrm{mM}$ phenylmethylsulfonyl fluoride, and the active guanosine-5' -triphosphate GTP-bound form of proteins was isolated and analyzed according to the manufacturer's instructions (Cell Signaling Technology). Cell lysates were briefly incubated with glutathione resin and glutathione-Stransferase fusion proteins, and GTP-bound proteins were eluted. The eluted samples were analyzed by Western blotting using antibodies against Ras, Rac1, and Cdc42 (Cell Signaling Technology).

\section{Western blotting}

MLE-12 cells were incubated with or without Slit2 and then collected at $15 \mathrm{~m}, 1 \mathrm{~h}$, and $6 \mathrm{~h}$ by centrifugation at $1200 \mathrm{rpm}$ for $5 \mathrm{~m}$ at $4{ }^{\circ} \mathrm{C}$.

Cells were lysed in RIPA buffer and quantified using the BCA assay. Protein samples were separated by sodium dodecyl sulfate-polyacrylamide gel electrophoresis and then transferred to a PVDF membrane. The transferred membranes were incubated at $4{ }^{\circ} \mathrm{C}$ overnight with 


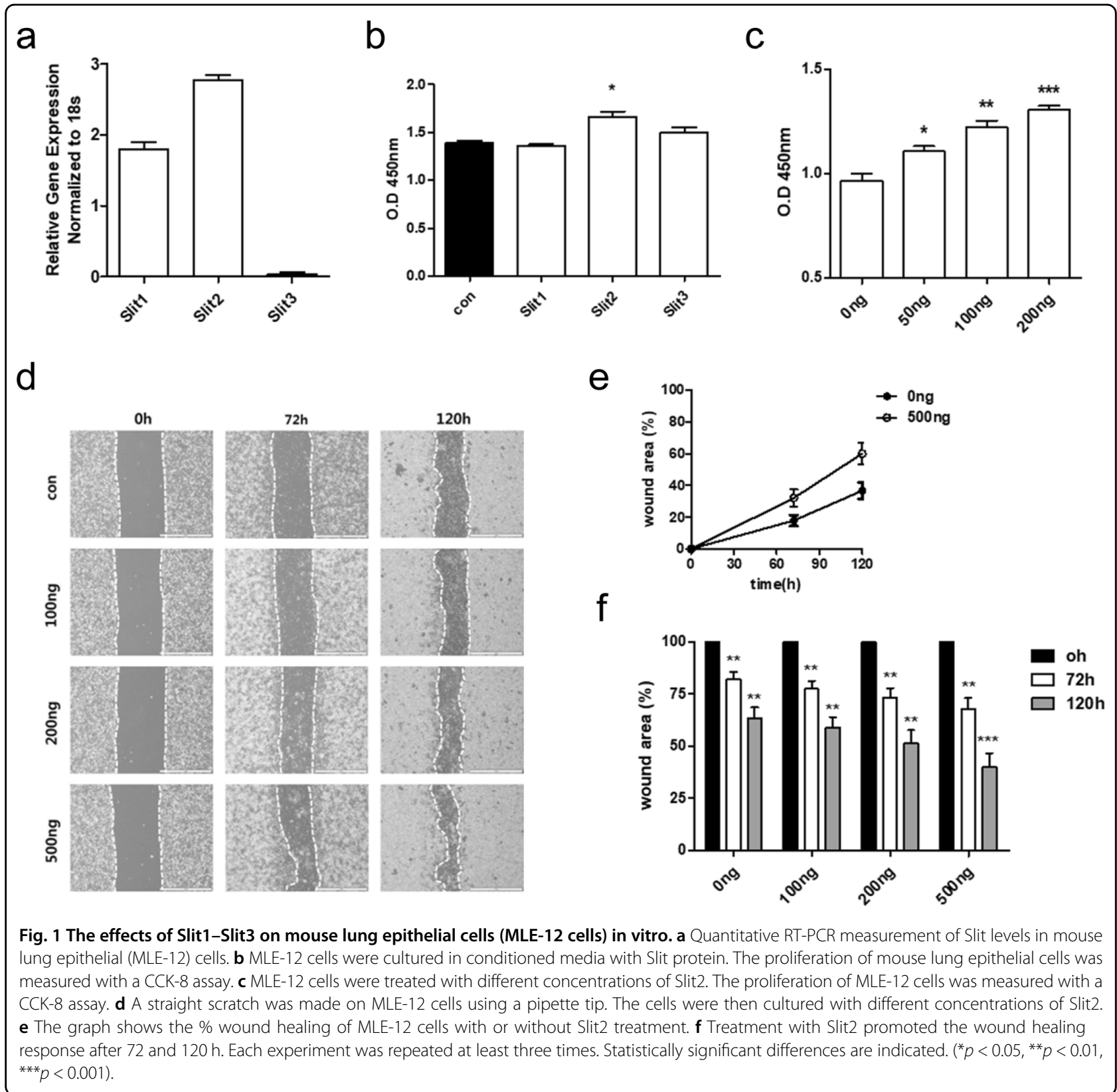

biotinylated primary antibodies against Akt, p-Akt, ERK, $\mathrm{p}$-ERK, and $\beta$-actin, incubated with HRP-conjugated secondary antibodies, and detected with a chemiluminescent membrane substrate.

\section{Results}

Slit2 improves the proliferation and migration of lung epithelial cells in vitro

First, we examined the expression pattern of Slit in MLE12 cells. Slit1 and Slit2 were expressed in MLE-12 cells, whereas Slit3 exhibited little or no expression (Fig. 1a). The protein levels of the Slit family showed similar expression patterns to those in the RT-PCR results (Supplemental Fig. 1). We then examined the effect of Slit 1-3 on MLE-12 cell proliferation in vitro using Slit as a ligand. When MLE12 cells were treated with each Slit isoform, MLE-12 cells treated with Slit2 exhibited significantly increased proliferation compared to those treated with the other isoforms (Fig. 1b). Next, when Slit2 was added at different concentrations, we found that cell proliferation increased in a concentration-dependent manner (Fig. 1c). The effect of Slit2 on MLE-12 cell migration in vitro was evaluated by using a scratch wound assay. The migration rates were determined by measuring wound closure at defined time 


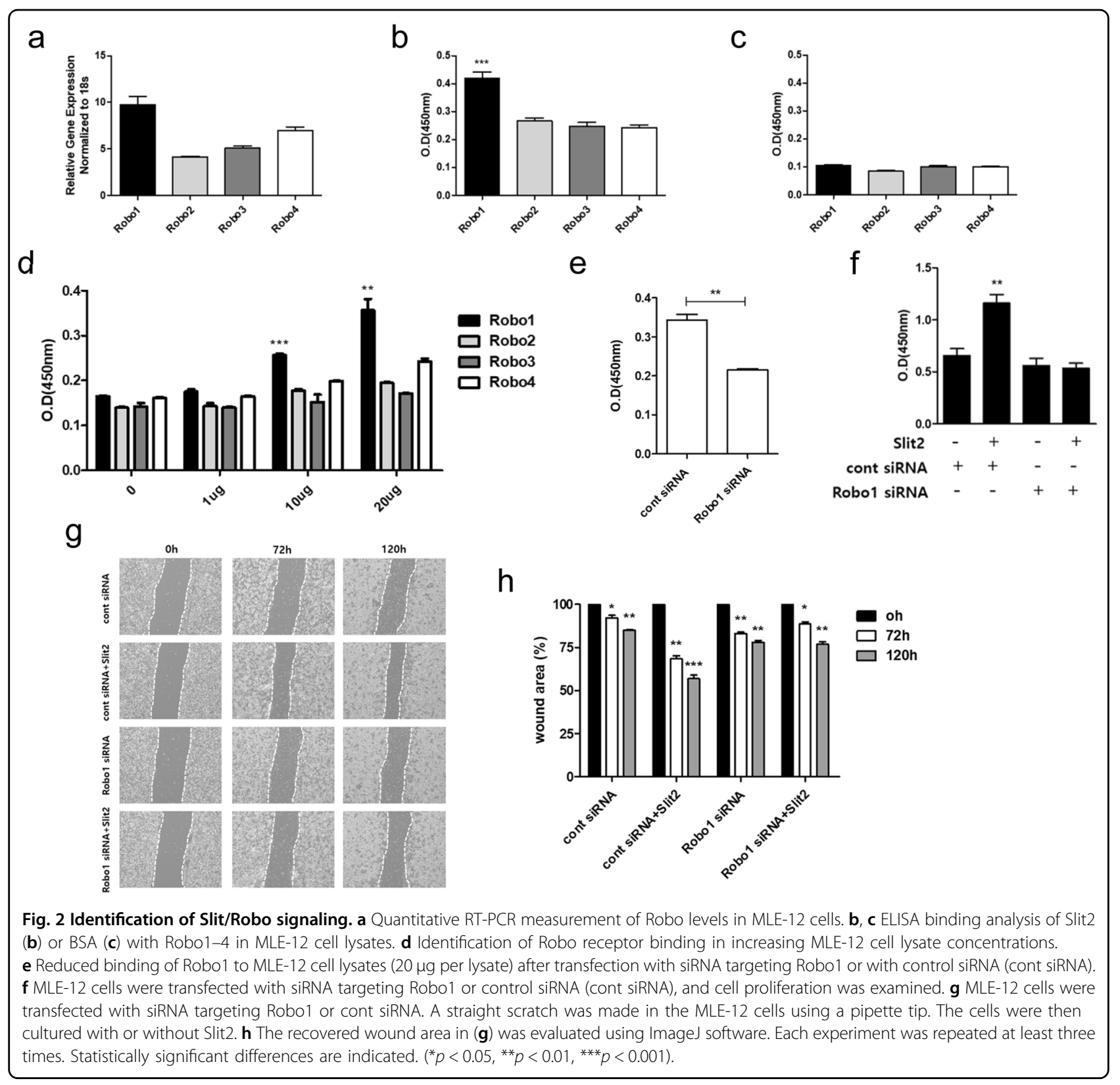

points in microscopy images (Fig. 1d). After treatment with Slit2, the recovered wound area confirmed that cell migration more than doubled, and Slit2 improved the movement of MLE-12 cells in a dose-dependent manner (Fig. 1e, f). These data confirmed the expression of Slit in MLE-12 cells, and cell proliferation and migration were increased by Slit 2 treatment.

\section{Expression of the Slit2 receptor and identification of receptor binding}

The Slit receptor includes four different types of Robo, and among them, the Robo receptor to which Slit2 preferentially binds was identified. To confirm the expression of Robo receptors, we examined the transcript level of Robo in MLE-12 cells. Overall, the expression of Robo1-4 in MLE-12 cells was confirmed, and the expression of Robo1 was more predominant than that of the others (Fig. 2a). In addition, it was confirmed that the protein level of Robo1 was the highest compared to that of the other proteins (Supplemental Fig. 1). These results suggest that Slit2 will preferentially bind to Robo1. ELISA indicated that the binding of Slit2 and Robo1 was the highest and confirmed that Slit2/Robo1 binding increased with increasing concentrations of MLE-12 cell lysate (Fig. 2b, d). However, BSA and MLE-12 cell lysates did not have a strong affinity for each other (Fig. 2c). 
a
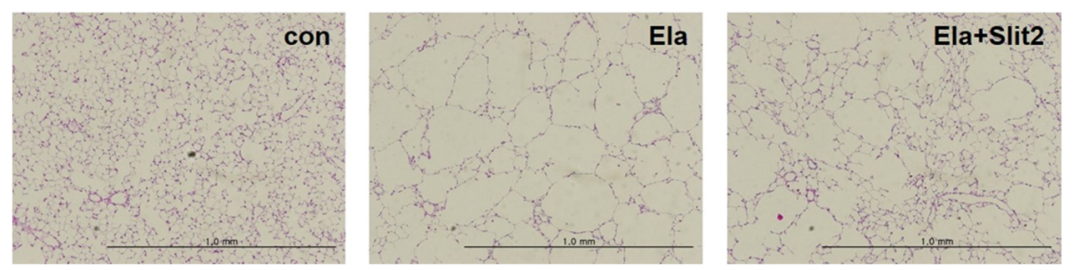

b

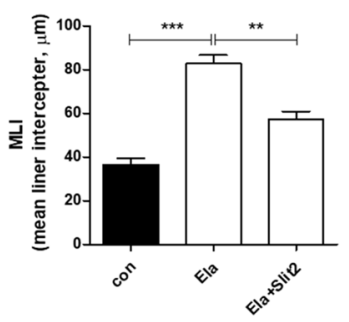

Fig. 3 The effects of Slit2 on lung regeneration in mice with elastase-induced emphysema. a C57BL/6 mice were intratracheally injected with elastase (Ela) on day 0 and then intranasally administered Slit2 (500 ng) on days 7-13. Hematoxylin and eosin (H\&E)-stained lung tissue sections on day 14. $\mathbf{b}$ Morphometric analysis of the mean linear intercept (MLI). The values represent the mean \pm standard error of the mean $(n=6)$. Statistically significant differences are indicated. ${ }^{* *} p<0.01,{ }^{* * *} p<0.001$ ).

To test the effect of Robo1 knockdown on MLE-12 cells, we applied Robo1 siRNA to MLE-12 cells and silenced the expression of Robo1. With the knockdown of Robo1, the binding of Slit2 to Robo1 in MLE-12 cell lysates was decreased compared to that in the group treated with the control siRNA (Fig. 2e). In MLE-12 cells with Robo1 knockdown, treatment with Slit2 did not affect cell proliferation (Fig. 2f). Furthermore, Robo1 knockdown decreased the migration of MLE-12 cells, as shown by the decrease in the recovered wound area (Fig. $2 g$, h). These results suggest that Slit2 preferentially binds to Robo1 and that Slit2/Robo1 signaling may have an important effect on the proliferation and migration of MLE-12 cells.

\section{Regenerative effects of Slit2 on an elastase-induced emphysema mouse model}

We confirmed that Slit2 is involved in MLE-12 cell proliferation and migration in vitro, and we tested the hypothesis that Slit2 plays a role in lung regeneration in the elastase-induced emphysema mouse model. After inducing emphysema with elastase, mice were treated by daily intranasal administration of Slit2 from days 7-13. To determine whether Slit2 restores elastase-induced lung destruction, lung tissue sections were stained with hematoxylin and eosin to analyze the histological changes in MLI counts. Alveolar damage and MLI increases were indicated in the group treated with elastase alone, and lung regeneration and MLI reductions were indicated in the group administered Slit2 after elastase injection (Fig. 3a, b). These data indicated that Slit2 administration may have a regenerative effect on the elastase-induced emphysema mouse model.

\section{Slit2 activates signaling pathways involved in cellular processes}

It is widely known that Slit/Robo signaling involves GTPases, which are small GTP-binding proteins that include the Ras superfamily: Ras, Rho, Rab, Arf, and $\operatorname{Ran}^{18-21}$. In addition, these proteins may be involved in regulatory mechanisms such as cell migration, angiogenesis, and neuronal morphogenesis ${ }^{22-24}$. We then examined whether Slit2 treatment increased the activity of GTPases in MLE-12 cells. Slit2 increased the levels of GTP-bound Ras but not the GTPases in the Rho family (Rac1 and Cdc42) (Fig. 4a). When bound to GTP, Ras is activated to stimulate multiple downstream targets, such as the ERK/MAP kinase pathway and PI3-kinase/Akt ${ }^{25-27}$. The ERK/MAP kinase and PI3-kinase/Akt pathways are major intracellular signaling pathways that are known to regulate various cellular processes, including cell proliferation, migration, and survival ${ }^{27-30}$. We, therefore, aimed to investigate whether ERK and Akt signal transduction was involved in Slit2-induced cell proliferation and migration. We examined the effect of Slit2 on the phosphorylation of ERK and Akt in MLE-12 cell lysates after Slit2 treatment. As shown in Fig. 4, Slit2 activated ERK and Akt phosphorylation. Phosphorylation increased within $15 \mathrm{~min}$ of Slit2 administration and peaked at $1 \mathrm{~h}$ (Fig. 4b). Densitometric analysis was performed to determine the ratio of phosphorylated to total ERK and Akt (Fig. 4c, d). When Robo1 was knocked down, there was no change in Ras activity or the phosphorylation of ERK and Akt by slit2 compared to the effects of control siRNA (Supplemental Fig. 2). 


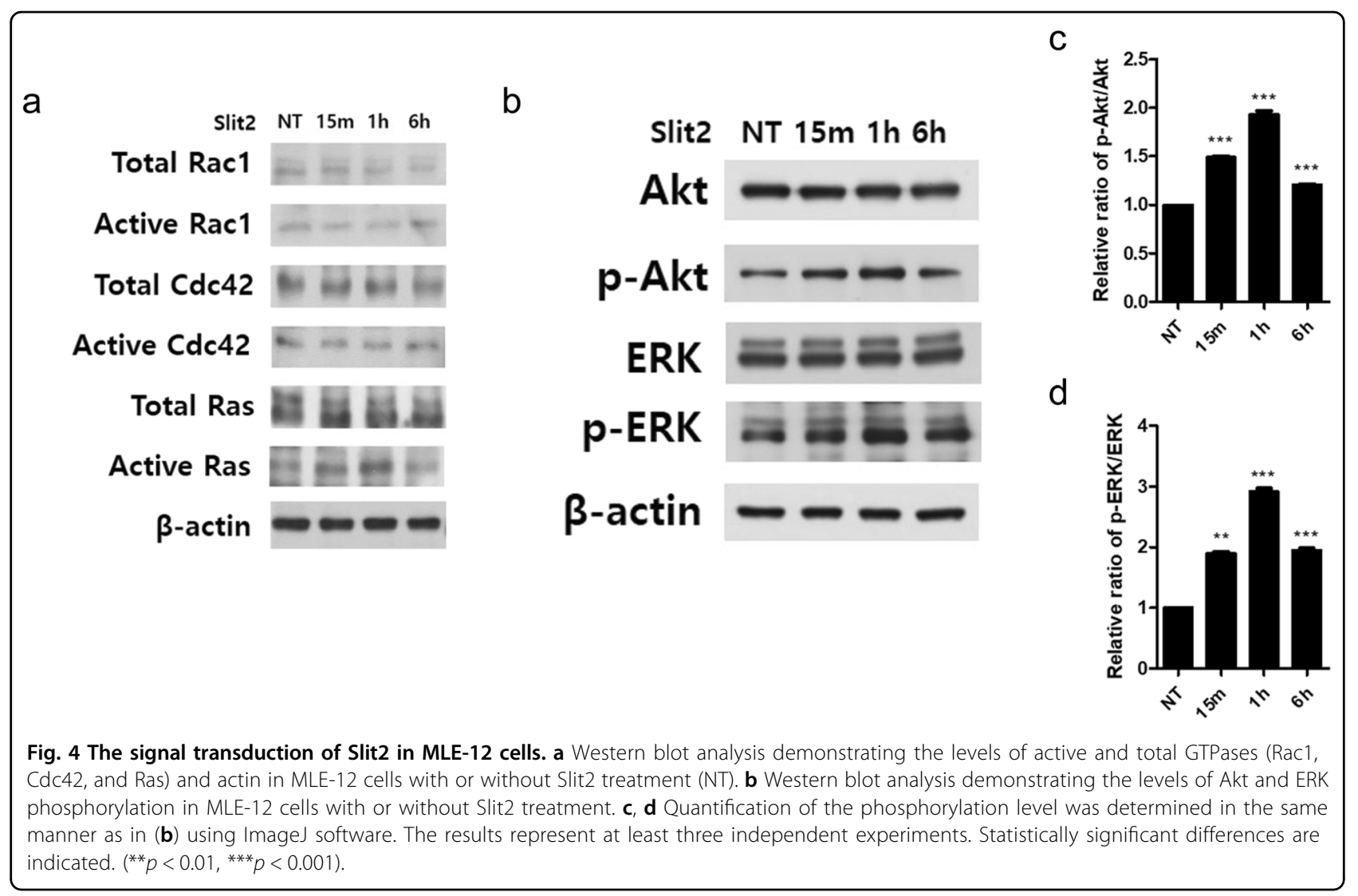

The results showed that Slit2-induced GTP-bound Ras increased phosphorylation of the downstream targets ERK and Akt and thus affected the proliferation and migration of MLE-12 cells.

\section{Discussion}

Recent studies have shown that Slit/Robo signaling has been implicated in cell proliferation, stem cell regulation, angiogenesis, and organ development ${ }^{4,6,8,9}$. Slit proteins are secreted glycoproteins with three isoforms; these proteins are involved in regulating the migration of axons and neurons during development ${ }^{13,14}$. Slit proteins bind to four Robo receptors (Robo1-Robo4), which are associated with downstream signaling that regulates various cellular processes ${ }^{11,31}$.

In this study, we examined the regenerative effect of Slit2 on cell proliferation and migration and an elastaseinduced emphysema mouse model. Our findings showed that Slit2 improved MLE-12 cell proliferation and migration, which was associated with Slit2 signaling through Robo1. In the elastase-induced emphysema mouse model, intranasal administration of Slit2 showed therapeutic effects on the regeneration of alveolar destruction. Slit2 treatment increased GTP-binding to Ras, which increased phosphorylation of the downstream targets ERK and Akt and may affect the proliferation and migration of MLE-12 cells. Research related to Slit has mainly focused on the nervous system, such as the regulation of axon and neuronal migration during development; however, Slit was recently confirmed to affect bone regeneration $^{32}$ and was found to play an important role. Slit/Robo signaling has also been shown to regulate the vascular system, including angiogenic processes such as endothelial cell migration and tube formation ${ }^{33}$.

With these studies as a foundation for our research, we present for the first time results that indicate that Slit2 may be effective for lung regeneration.

There is currently no standard treatment regimen for COPD; it is, therefore, vital to develop a therapeutic agent, and Slit2 shows potential as a treatment candidate based on its regenerative effects. Here, we first focused on the proliferation of alveolar epithelial cells in vitro and found that Slit2 could increase the proliferation of these cells. Slit and Robo each have several isoforms. Our data showed that compared with the other Slit proteins, Slit2 increased the proliferation and migration of MLE-12 cells, and of the Robo protein family, Robo1 preferentially bound to Slit2.

In addition, when Robol was knocked down using Robo1 siRNA, the proliferation of MLE-12 cells did not increase in response to Slit2 treatment, suggesting that Slit2/Robo1 signaling may play an important role in lung regeneration. 
Next, based on the in vitro results, we investigated whether Slit2 affected lung regeneration.

Recently, there has been an effort to develop a new drug for bone regeneration that targets Slit, which is an important mechanism for bone regeneration ${ }^{32}$. On the other hand, considering that Slit is also present in the lungs, based on the in vitro results, we aimed to confirm whether Slit2 could act on emphysema-associated lung regeneration. When the mouse emphysema model was induced with elastase and intranasally administered Slit2, the therapeutic effects on the repair of alveolar damage were shown. Furthermore, among GTPases, Ras-mediated activation of ERK and Akt was increased by increasing Ras activity through Slit2/Robo signaling.

In summary, these factors may play critical roles in epithelial cell proliferation, facilitating the repair of the damaged epithelium associated with lung regeneration.

Our findings suggest for the first time that Slit2 may have potential as a therapeutic drug candidate for the treatment of emphysema-associated lung regeneration.

\section{Acknowledgements}

We thank the members of the Asan Medical Center Animal Facility for their technical expertise. This research was supported by the Basic Science Research Program through the National Research Foundation of Korea (NRF) funded by the Ministry of Education (NRF-2018R1A2B2001065).

\section{Author details}

${ }^{1}$ Asan Institute for Life Sciences, Seoul, Korea. ${ }^{2}$ Department of Internal Medicine, University of Ulsan College of Medicine, Seoul, Korea. ${ }^{3}$ Department of Pulmonary and Critical Care Medicine, Asan Medical Center, Seoul, Korea

\section{Conflict of interest}

The authors declare no competing interests.

\section{Publisher's note}

Springer Nature remains neutral with regard to jurisdictional claims in published maps and institutional affiliations.

Supplementary information The online version contains supplementary material available at https://doi.org/10.1038/s12276-021-00633-8.

Received: 6 November 2020 Revised: 23 March 2021 Accepted: 5 April 2021.

Published online: 25 May 2021

\section{References}

1. Rabe, K. F. et al. Global strategy for the diagnosis, management, and prevention of chronic obstructive pulmonary disease: GOLD executive summary. Am. J. Respir. Crit. Care Med. 176, 532-555 (2007).

2. Mannino, D. M. \& Buist, A. S. Global burden of COPD: risk factors, prevalence, and future trends. Lancet 370, 765-773 (2007).

3. Rothberg, J. M., Jacobs, J. R., Goodman, C. S. \& Artavanis-Tsakonas, S. Slit: an extracellular protein necessary for development of midline glia and commissural axon pathways contains both EGF and LRR domains. Genes Dev. 4, 2169-2187 (1990).

4. Rothberg, J. M., Hartley, D. A., Walther, Z. \& Artavanis-Tsakonas, S. Slit: an EGFhomologous locus of $D$. melanogaster involved in the development of the embryonic central nervous system. Cell 55, 1047-1059 (1988).
5. Ypsilanti, A. R., Zagar, Y. \& Chédotal, A. Moving away from the midline: new developments for Slit and Robo. Development 137, 1939-1952 (2010).

6. Borrell, $V$. et al. Slit/Robo signaling modulates the proliferation of central nervous system progenitors. Neuron 76, 338-352 (2012).

7. Ballard, M. S. et al. Mammary stem cell self-renewal is regulated by Slit2/ Robo1 signaling through SNAl1 and mINSC. Cell Rep. 13, 290-301 (2015).

8. Bedell, V. M. et al. roundabout4 is essential for angiogenesis in vivo. Proc. Natl Acad. Sci. USA 102, 6373-6378 (2005).

9. Greenberg, J. M., Thompson, F. Y., Brooks, S. K., Shannon, J. M. \& Akeson, A. L. Slit and robo expression in the developing mouse lung. Dev. Dyn. 230 350-360 (2004).

10. Dickinson, R. E. \& Duncan, W. C. The SLIT-ROBO pathway: a regulator of cell function with implications for the reproductive system. Reproduction 139 697-704 (2010)

11. Blockus, H. \& Chédotal, A. Slit-robo signaling. Development 143, 3037-3044 (2016).

12. Mehlen, P., Delloye-Bourgeois, C. \& Chédotal, A. Novel roles for Slits and netrins: axon guidance cues as anticancer targets? Nat. Rev. Cancer 11, 188-197 (2011).

13. Morlot, C. et al. Structural insights into the Slit-Robo complex. Proc. Natl Acad. Sci. USA 104, 14923-14928 (2007).

14. Hohenester, E. Structural Insight into Slit-Robo Signalling. (Portland Press Ltd., 2008).

15. Liu, Z. et al. Extracellular lg domains 1 and 2 of Robo are important for ligand (Slit) binding. Mol. Cell. Neurosci. 26, 232-240 (2004).

16. Kim, Y.-S. et al. Tracking intravenous adipose-derived mesenchymal stem cells in a model of elastase-induced emphysema. Tuberc. Respir. Dis. 77, 116-123 (2014).

17. Thurlbeck, W. M. Measurement of pulmonary emphysema. Am. Rev. Respir. Dis. 95, 752-764 (1967).

18. Colicelli, J. Human RAS superfamily proteins and related GTPases. Sci. STKE 2004, re13-re13 (2004).

19. Buday, L. \& Downward, J. Many faces of Ras activation. Biochim. Biophys. Acta 1786, 178-187 (2008).

20. Mitin, N., Rossman, K. L. \& Der, C. J. Signaling interplay in Ras superfamily function. Curr. Biol. 15, R563-R574 (2005).

21. Mor, A., Dustin, M. L. \& Philips, M. R. Small GTPases and LFA-1 reciprocally modulate adhesion and signaling. Immunol. Rev. 218, 114-125 (2007).

22. Chaturvedi, L. S., Marsh, H. M. \& Basson, M. D. Role of RhoA and its effectors ROCK and mDia1 in the modulation of deformation-induced FAK, ERK, p38, and MLC motogenic signals in human Caco-2 intestinal epithelial cells. Am. J. Physiol. Cell Physiol. 301, C1224-C1238 (2011)

23. Rama, N. et al. Slit2 signaling through Robo1 and Robo2 is required for retinal neovascularization. Nat. Med. 21, 483 (2015)

24. Luo, L. Rho GTPases in neuronal morphogenesis. Nat. Rev. Neurosci. 1, 173-180 (2000).

25. Kolch, W. Coordinating ERK/MAPK signalling through scaffolds and inhibitors. Nat. Rev. Mol. Cell Biol. 6, 827-837 (2005).

26. Murphy, L. O. \& Blenis, J. MAPK signal specificity: the right place at the right time. Trends Biochem. Sci. 31, 268-275 (2006).

27. Shaw, R. J. \& Cantley, L. C. Ras, PI (3) K and mTOR signalling controls tumour cell growth. Nature 441, 424-430 (2006).

28. Pratsinis, H. \& Kletsas, D. PDGF, bFGF and IGF-I stimulate the proliferation of intervertebral disc cells in vitro via the activation of the ERK and Akt signaling pathways. Eur. Spine J. 16, 1858-1866 (2007).

29. Fournier, N. M., Lee, B., Banasr, M., Elsayed, M. \& Duman, R. S. Vascular endothelial growth factor regulates adult hippocampal cell proliferation through MEKJERK-and PI3K/Akt-dependent signaling. Neuropharmacology 63, 642-652 (2012).

30. Park, J. H. \& Han, H. J. Caveolin-1 plays important role in EGF-induced migration and proliferation of mouse embryonic stem cells: involvement of PI3KJAkt and ERK. Am. J. Physiol. Cell Physiol. 297, C935-C944 (2009).

31. Tong, M., Jun, T., Nie, Y., Hao, J. \& Fan, D. The role of the Slit/Robo signaling pathway. J. Cancer 10, 2694 (2019).

32. Koh, J.-M. Osteoclast-derived SLIT3 is a coupling factor linking bone resorption to bone formation. BMB Rep. 51, 263 (2018).

33. Yuen, D. A. \& Robinson, L. A. Slit2-Robo signaling: a novel regulator of vascular injury. Curr. Opin. Nephrol. Hypertens. 22, 445-451 (2013). 